\title{
COMPORTAMENTO ALIMENTAR E DIETA DA "FALSA-CORAL" OXYRHOPUS GUIBEI HOGE \& ROMANO (SERPENTES, COLUBRIDAE) ${ }^{1}$
}

\author{
Rogério de Oliveira Andrade ${ }^{2}$ \\ Renato A. Matias Silvano ${ }^{2}$
}

\begin{abstract}
FEEDING BEHAVIOR AND DIET OF THE OXYRHOPUS GUIBEI HOGE \& ROMANO (SERPENTES, COLUBRIDAE). The feeding behavior and constriction pattern of adults and juveniles Oxyrhopus guibei Hoge \& Romano, 1978 preying on rodents and lizards are described. An analysis of stomach contents of snakes preserved in collections was made to study its diet. The results indicate that this snake constricts prey with right to left side movements, horizontal position and with the dorsal portion of its body. Rodents were constricted by adult snakes whereas lizard were swallowed alive. The diet is composed of lizards and rodents, with an ontogenetic shift from lizards to rodents.
\end{abstract}

KEY WORDS. Serpentes, Colubridae, Oxyrhopus guibei, feeding behavior

Serpentes são predadores que apresentam grande variedade de hábitos alimentares (DITMARS 1912; MUSHINSKI 1987). Entre os métodos de captura e subjugação de presas por serpentes encontram-se o bote simples, bote e envolvimento da presa com uma volta do corpo, envenenamento e constrição (DITMARS 1912). Algumas serpentes podem utilizar mais de um mecanismo de subjugação, como por exemplo, constrição e envenenamento (SHINE \& SCHWANER 1985).

Serpentes da família Colubridae apresentam variados padrões de constrição, ao passo que as famílias mais primitivas, como Acrochordidae, Aniliidae, Boidae e Xenopeltidae, utilizam um único padrão de constrição (GREENE \& BURGHARDT 1978). Durante a constrição, a presa é imobilizada pela pressão exercida por dois ou mais pontos do corpo da serpente (GREENE \& BURGHARDT 1978). Três categorias básicas de constrição foram propostas por WILLARD (1977): a) a serpente aplica as espiras do corpo com o ventre em direção à própria cabeça (método 1); b) a serpente aplica as espiras do corpo com o dorso virado em direção à própria cabeça (método 2); c) a serpente aplica as espiras de modo irregular (por vezes sobrepondo-as), sem uma única face em contato com a presa (método 3). Alguns estudos enfocam a dominância de lado na direção do movimento do corpo durante a constrição por serpentes, sendo consideradas dextrógiras as serpentes que realizam o movimento de constrição da direita para a esquerda e levógiras as que realizam o

1) Com auxilio do Fundo de Apoio ao Ensino e à Pesquisa (FAEP), Universidade Estadual de Campinas.

2) Departamento de Zoologia, Instituto de Biologia, Universidade Estadual de Campinas. Caixa Postal 6109, 13083-970 Campinas, São Paulo, Brasil. 
processo inverso (HEINRICH \& KLASSEN 1985; LOPES et al. 1991). Não há consenso sobre o quanto a dominância de lado representa uma característica de variação específica ou individual em serpentes (HEINRICH \& KLASSEN 1985; LOPES et al. 1991).

Estudos de dieta em serpentes são realizados principalmente através da análise do tubo digestivo de animais preservados (MUSHINSKI 1987). Para estabelecer os itens da dieta é desejável o exame de grande número de indivíduos (SHINE \& WEBB 1990; MARQUES \& PUORTO 1994). Informações sobre dieta de serpentes neotropicais são escassas (MUSHINSKI 1987), havendo algumas informações sobre as espécies brasileiras (AMARAL 1977; CUNHA \& NASCIMENTO 1978; LEMA et al. 1983; SAZIMA \& HADDAD 1992).

A "falsa-coral" Oxyrhopus guibei Hoge \& Romano, 1978 (à respeito da situação taxonômica, ver ZAHER \& CARAMASCHI 1992) é uma serpente terrícola, de áreas abertas, com atividade predominantemente crepuscular e noturna (SAZIMA \& ABE 1991; SAZIMA \& HADDAD 1992). Essa serpente possui ampla distribuição pelos Estados de Mato Grosso, Paraná, São Paulo e Minas Gerais, neste último ocorrendo simpatricamente com O. trigeminus Duméril, Bibron \& Duméril, 1854 na região de Lagoa Santa (HogE \& ROMANO 1976). Oxyrhopus guibei alimenta-se de roedores e lagartos, que subjuga por constrição (SAZIMA \& MARTINS 1990; SAZIMA \& HADDAD 1992). SAZIMA \& ABE (1991) encontraram roedores murídeos, lagartos e um filhote de pomba como parte da dieta. No presente estudo descreve-se o comportamento alimentar e analisa-se os itens da dieta de $O$. guibei. Comenta-se as diferenças comportamentais que ocorrem entre jovens e adultos, frente a diferentes tipos de presa, e a ocorrência de dominância de lado durante o movimento de constrição da presa.

\section{MATERIAL E MÉTODOS}

Uma vez que os hábitos das serpentes podem variar regionalmente (MUSHINSKI 1987; STRUSSMANN \& SAZIMA 1993), limitamos a região de estudo. As serpentes estudadas provém de região limitada ao norte pela Serra da Mantiqueira (região dos municípios de Moji Mirim e São João da Boa Vista), a sudeste com a Serra do Mar, a sudoeste com a Serra de Botucatu e a noroeste com a região dos cerrados de São Carlos e Araraquara.

Nas análises de comportamento alimentar empregaram-se os padrões propostos por GREENE \& BURGHARDT (1978) e WILLARD (1977). Observou-se exemplares cativos adultos (serpentes medindo mais de $40 \mathrm{~cm}$ de comprimento total) e juvenis (serpentes medindo menos de $40 \mathrm{~cm}$ de comprimento total) de $O$. guibei, procurando minimizar as influências externas (LEHNER 1979), em períodos diurno e noturno. Adotou-se os métodos de "todas as ocorrências" e "amostragem de sequências" (LeHNER 1979; MARQues \& PUORTO 1994; SAZIMA 1989). Como alimento, foram oferecidas lagartixas, Hemidactylus mabouia (Moreau de Jonnés, 1818), e camundongos de laboratório, Mus musculus Linnaeus, 1766, registrando o comportamento da serpente frente à presa, desde a captura até o final da ingestão (cf. SAZIMA 1989). Em caso de rejeição da presa, esta foi retirada após um período de dez minutos. Utilizou-se um total de vinte serpentes, doadas pelo Instituto 
Butantan, Zoológico de Sorocaba ou coletadas na região de Campinas.

No estudo de dieta, examinou-se 250 exemplares (dos quais foram retirados 30 conteúdos do tubo digestivo), preservados nas coleções herpetológicas do Instituto Butantan (IB) e do Museu de História Natural da Universidade Estadual de Campinas (ZUEC). Utilizou-se coleções de referência de mamíferos e répteis visando a identificação dos itens encontrados no tubo digestivo.

\section{RESULTADOS}

Na fase de detecção e localização de presas, Oxyrhopus guibei quase prontamente persegue roedores à medida em que estes se movem. Nove serpentes (seis adultos e três juvenis) alimentaram-se das presas oferecidas, sendo que alguns indivíduos chegaram a se alimentar seis vezes e outros apenas uma vez. As serpentes que se alimentaram de lagartixas (Hemidactylus mabouia) apresentaram dificuldade em localizar esta presa imóvel e camuflada. Registramos um total de 33 observações do comportamento alimentar. Seis adultos alimentaram-se de roedores $(\mathrm{n}=26)$, três adultos alimentaram-se de lagartos $(n=3)$ e três juvenis alimentaram-se de lagartos $(n=4)$.

Adultos frente a roedores localizam a presa imediatamente após esta ser colocada no terrário, desferindo bote contra a parte anterior $(\mathrm{n}=13)$, ou posterior $(n=9)$ do corpo do roedor e raramente abocando o meio do corpo da presa. $\mathrm{Na}$ sequência do bote a serpente constringe o roedor com movimento dextrógiro, utilizando-se do método 2 de constrição, na posição horizontal (Fig. 1, Tab. I). Adultos alimentando-se de roedores demonstraram tendência a constringir realizando movimento dextrógiro (23:3), aplicando o método 2 (22:4) na posição horizontal (23:3) (Tab. I). Após a morte da presa, a serpente afrouxa as espiras e procura a porção anterior, tocando com as regiões ventral e lateral da cabeça os pelos do roedor. Uma vez encontrada a região anterior, a serpente inicia a ingestão pela cabeça da presa, com movimentos alternados das maxilas, com auxílio $(\mathrm{n}=15)$ ou não $(n=7)$ das espiras do corpo. Os adultos que se alimentaram de lagartos não utilizaram a constrição, abocando a presa pela porção anterior $(\mathrm{n}=2)$ ou posterior $(n=1)$, ingerindo-a viva a partir da cabeça, utilizando $(n=1)$ ou não $(n=2)$ as espiras do corpo.

Os juvenis levaram certo tempo para perceber os lagartos, desferindo bote contra a sua região anterior $(n=2)$ ou posterior $(n=2)$, constringindo imediatamente com movimento dextrógiro $(n=3)$ ou levógiro $(n=1)$. Os lagartos foram ingeridos a partir da cabeça, com $(n=3)$ ou sem $(n=1)$ auxílio das espiras do corpo. Um dos juvenis ingeriu um lagarto a partir da cauda.

$\mathrm{Na}$ análise de 30 conteúdos do tubo digestivo, foram encontrados pelos e restos semi-digeridos de roedores murídeos $(\mathrm{n}=26)$. Também encontrou-se três espécies de lagartos: Hemidactylus mabouia $(\mathrm{n}=2)$, Mabuya frenata Gallardo, 1968 $(\mathrm{n}=1)$ e Tropidurus itambere Rodrigues, $1987(\mathrm{n}=1)$. Em serpentes medindo menos de $40 \mathrm{~cm}$ de comprimento total (juvenis), observou-se apenas lagartos como conteúdo do tubo digestivo $(\mathrm{n}=3)$. Em serpentes maiores de $40 \mathrm{~cm}$ (adultos), ocorreram roedores $(\mathrm{n}=26)$, com exceção de uma serpente de $80 \mathrm{~cm}$ que apresentou um lagarto como conteúdo estomacal. 
Tabela I. Três caracteristicas da constriç̧ão em Oxyrhopus guibei, ao se alimentar de roedores. Os números referem-se à quantidade de observações em cada categoria (número de eventos predatórios observados $=26$ ).

\begin{tabular}{ccccccccc}
\hline \multicolumn{2}{c}{ Movimento } & & \multicolumn{2}{c}{ Tipo } & & \multicolumn{2}{c}{ Posição } \\
\cline { 1 - 2 } \cline { 7 - 8 } Dextrógiro & Levógiro & & Método 2 & Método 3 & & Horizontal & Vertical \\
\hline 23 & 3 & 22 & 4 & 23 & 3 \\
\hline
\end{tabular}

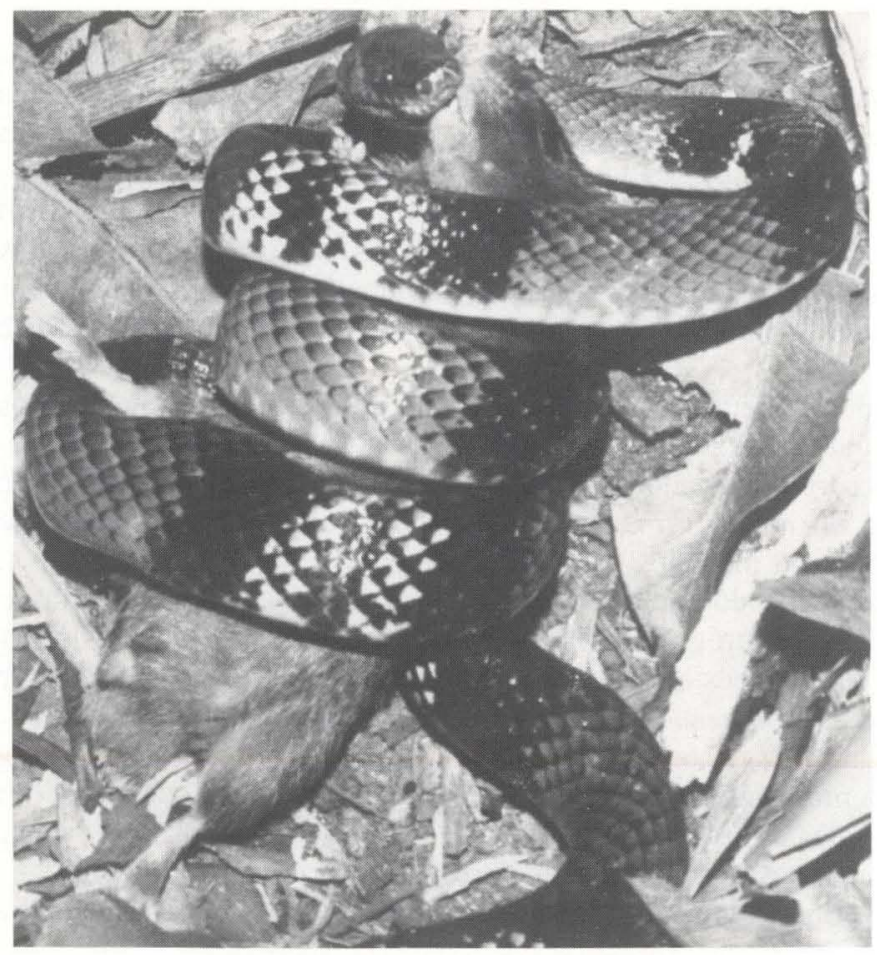

Fig. 1. Indivíduo adulto de Oxyrhopus guibei, constringindo roedor com movimento dextrógiro, em posição horizontal e empregando o método 2 (cf. WILLARD 1977).

\section{DISCUSSÃO}

De acordo com KARDONG (1993), roedores de laboratório oferecidos à cascavel, Crotalus viridis oreganus Holbrook, 1840 eliciam comportamento predatório equivalente ao demonstrado por serpentes apresando roedores na natureza. Assim, o fato de utilizar roedores de laboratório não deve ter interferido de maneira acentuada nos resultados obtidos [ver também SAZIMA (1989) para Bothrops jararaca (Wied, 1824)].

Além do olfato, a percepção visual em $O$. guibei parece exercer importante papel na captura de presas, apesar de seus hábitos predominantemente noturnos 
(SAZIMA \& HADDAD 1992). Com relação à captura de roedores, os indivíduos não demonstraram preferência relacionada à abocar a região anterior ou posterior da presa. Entretanto, as presas oferecidas raramente foram abocadas pelo meio do corpo, devido a este modo de captura dificultar a aplicação das espiras de constrição. Segundo Moori (1991), a serpente Elaphe quadrivirgata (Boie, 1826) da família Colubridae apresenta uma tendência a capturar roedores pela porção anterior do corpo, indicando que este comportamento possa diminuir a possibilidade de retaliação por parte da presa. Em $O$. guibei não encontrou-se tal tendência, fato talvez relacionado à presença de veneno nesta espécie, uma vez que o veneno poderia auxiliar na subjugação (BAILEY 1966; SAZIMA \& HADDAD 1992).

Quando utilizou constrição, $O$. guibei efetuou movimento dextrógiro na maior parte das vezes (Tab. I), mantendo portanto o lado esquerdo do corpo em contato com a presa (Fig. 1), indicando dominância de lado a nível específico (veja também figura em SAZIMA \& HADDAD 1992). Estudos com serpentes Boídeas, realizados por LOPES et al. (1991), demonstraram dominância de lado a nível individual e em baixa freqüência. Quanto ao método de constrição, $O$. guibei utilizou principalmente o método 2 , com o dorso voltado em direção à cabeça. $\mathrm{O}$ fato de $O$. guibei ter empregado por vezes o método 3 relaciona-se à falta de espaço para a constrição, feita próximo a algum tipo de obstáculo, ou devido ao fato da serpente capturar a presa pelo meio do corpo, dificultando a formação das espiras. Nossos resultados estão de acordo com as observações de WiLlaRd (1977), que analisou o comportamento de constrição em 95 espécies de serpentes pertencentes às famílias Boidae e Colubridae, demonstrando que a maioria dos colubrídeos utiliza o método 2, sendo o método 3 utilizado por colubrídeos que constringiram as presas em espaço confinado. Houve uma nítida preferência pela posição horizontal no ato da constrição (Fig. 1, Tab. I), embora GREENE \& BURGHARDT (1978) considere este parâmetro muito variável. O mesmo autor encontrou variações intergenéricas, interespecíficas e em alguns casos individuais relacionadas ao movimento de aplicação das espiras efetuado por 27 espécies de colubrideos, argumentando que a constrição tenha evoluido independentemente e repetidas vezes nesta família de serpentes (GREENE \& BURGHARDT 1978).

$\mathrm{Na}$ quase totalidade das observações, os indivíduos de $O$. guibei iniciaram a ingestão a partir da cabeça da presa. Este comportamento de ingestão diminui a resistência imposta pelos apêndices da presa, reduzindo assim o tempo e a energia dispendidos durante a sequência alimentar (SAZIMA 1989; MOORI 1991).

Observou-se variações no comportamento alimentar de $O$. guibei frente a diferentes presas. Roedores sempre foram constringidos e ingeridos, depois de mortos, ao passo que lagartos foram ingeridos vivos e sem constrição por serpentes adultas, embora tenham sido constringidos e ingeridos, depois de mortos por juvenis. Predominância de uso de constrição em roedores também foi registrada por MOORI (1991) para Elaphe quadrivirgata, estando associada a maior atividade e periculosidade deste tipo de presa, ao passo que a ausência de constrição de lagartos (presas menos ativas e perigosas), minimizaria os gastos de tempo e energia no decorrer da sequência alimentar. O fato de somente juvenis de $O$. guibei constringirem lagartos pode estar relacionado ao maior tamanho e periculosidade deste tipo 
de presa para esta classe de tamanho de serpentes. As presas tendem a ser constringidas à medida em que aumenta o seu tamanho e periculosidade, conforme verificado para os colubrideos Elaphe quadrivirgata (Moori 1991) e Pituophis melanoleucus (Daudin, 1842) (DE QUEIROZ 1984).

Os gêneros Clelia Fitzinger, 1826 e Pseudoboa Schneider, 1801 são filogeneticamente próximos a Oxyrhopus Wagler, 1830, na tribo Pseudoboini Jenner \& Dowling, 1985. Clelia rustica (Cope, 1878) e C. occipitolutea (Duméril, Bibron and Duméril, 1854) também constringem a presa com movimento dextrógiro (Costa-PInTo 1993). Em Siphlophis pulcher (Raddi, 1820), outra espécie de Pseudoboini, também foi verificada constrição dextrógira, indicando uma tendência neste grupo de serpentes (SAZIMA \& ARGÔLO 1994).

SAZIMA \& ABE (1991) registraram a ocorrência dos roedores murídeos, Bolomys lasiurus (Lundi, 1841) ( $\mathrm{n}=2)$, Calomys laucha (Desmarest, 1819) $(\mathrm{n}=1)$, Mus musculus $(\mathrm{n}=1)$ e Rattus sp $(\mathrm{n}=1)$; do lagarto iguanídeo Tropidurus itambere Rodrigues, 1987, e de filhote da pomba juriti Leptotila sp. Os nossos resultados, assim como os de SAZIMA \& ABE (1991), indicam que a dieta de O. guibei é composta principalmente de roedores e lagartos. Um item da dieta desta serpente ainda não descrito na literatura, o lagarto Mabuya frenata (Cope, 1862), é uma presa de difícil apreensão por apresentar tegumento duro e liso (SHINE \& SCHWANER 1985). Não observou-se aves em nossas análises de conteúdo, sendo que o filhote de juriti (Columbidae) registrado por SAZIMA \& ABE (1991), provavelmente constitui um item ocasional na dieta desta serpente predominantemente terrícola. Ocorre uma mudança ontogenética na dieta de $O$. guibei, passando de lagartos para roedores, à medida que a serpente cresce. Mudança de dieta foi observada também em Oxyrhopus clathratus Duméril, Bibron and Duméril, 1854 e $O$. digitalis (Reuss, 1834) (I. Sazima, com. pess.). A dieta de alguns viperídeos e colubrídeos varia ontogeneticamente (MUSHINSKI 1987; SAZIMA 1992; MCCOY \& CENSKY 1992; MARQUES \& PUORTO 1994). A análise de conteúdos estomacais de Porthidium yucatanicum (Smith, 1941) (Viperidae), mostrou que a proporção de lagartos é a mesma na dieta de juvenis e adultos, porém somente os segundos apresentam roedores (MCCOY \& CENSKY 1992). Adultos de Erythrolamprus aesculapii (Linnaeus, 1766) alimentam-se somente de serpentes da família Colubridae, ao passo que os juvenis, além de serpentes, apresam lagartos gimnoftalmídeos (MARQUES \& PUORTO 1994). A mudança ontogenética pode ocorrer devido à ampliação no espectro de presas com o crescimento da serpente (MCCOY \& CENSKY 1992), uma menor disponibilidade de presas de tamanho adequado para a fase juvenil (SAZIMA \& MARTINS 1990; MARQUES \& PUORTO 1994), ou à periculosidade da presa, uma vez que tanto no caso de $O$. guibei, como no das demais espécies mencionadas, os adultos tendem a se alimentar de presas mais perigosas.

O comportamento alimentar e a dieta indicam que $O$. guibei é uma serpente que apresenta o comportamento de constrição bem desenvolvido, sendo eficiente predadora de roedores. A preferência por áreas abertas (SAZIMA \& ABE 1991; SAZIMA \& HADDAD 1992), aliada à dieta composta principalmente por animais que sobrevivem bem em ambientes urbanos (roedores murídeos e lagartixas), indicam que esteja adaptada a áreas antropicamente perturbadas (PUORTO et al. 1991). 
AGRADECIMENTOS. A Ivan Sazima, pela orientação, incentivo e leitura crítica do manuscrito. A Paulo R. Manzani, José P. Pombal Jr. e funcionários do Museu de História Natural e do Departamento de Zoologia da UNICAMP, pelo apoio. A Sérgio Rangel, do Zoológico de Sorocaba e a Giuseppe Puorto e demais pesquisadores do Instituto Butantan pelo apoio e fornecimento do material para estudo. A Otávio A. V. Marques, pelo incentivo e leitura crítica do manuscrito. À FAEP (UNICAMP), pelo auxílio financeiro (processo 0834/93, fase 30).

\section{REFERÊNCIAS}

AMARAL, A. 1977. Serpentes do Brasil, iconografia colorida. São Paulo, Melhoramentos \& Edusp, $1^{\mathrm{a}}$ ed, 246p.

BAILEY, J.R. 1966. Modes of evolution in New World opistoglyph snakes. Mem. Inst. Butantan 33: 67-72.

Costa-Pinto, C. 1993. Constrição na subjugação de presas por duas espécies de Clelia (Serpentes: Colubridae). Resumos do III Congresso Latino Americano de Herpetologia, Campinas, p.178.

Cunha, O.R. \& F.P. NAscimento. 1978. Ofídios da Amazônia. X - As cobras da região leste do Pará. Publ. Avuls. Mus. Paraense Emílio Goeldi 31: 1-218.

DE QueIROZ, A. 1984. Effects of prey type on prey handling behavior of the bullsnake, Pituophis melanoleucus. J. Herpetol. 18 (3): 333-336.

DITMARS, R.L. 1912. The feeding habits of serpents. Zoologica 1: 197-238.

GREENE, H.W. \& G.M. BuRGHARDT. 1978. Behavior and phylogeny: constriction in ancient and modern snakes. Science 200: 74-77.

HeINRICH, M.L. \& H.E. KLASSE. 1985. Side dominance in constricting snakes. J. Herpetol. 19: 531-533.

Hoge, A.R. \& S.A.R.W.L. Romano. 1976. Description of a new subspecies of Oxyrhopus Wagler (Serpentes, Colubridae). Mem. Inst. Butantan 40/41: 55-62.

JenneR, J.V. \& H.G. Dowling. 1985. Taxonomy of american Xenodontinae snakes: the tribe Pseudoboini. Herpetologica 41 (2): 161-172.

KARDONG, K.V. 1993. The predatory behavior of the northern pacific ratlesnake (Crotalus viridis oreganus): laboratory versus wild mice as prey. Herpetologica 49 (4): 457-463.

LEHNER, P.N. 1979. Handbook of ethological methods. New York, Garland S.T.P.M., 403p.

Lema, T. DE; M.L. de Araújo; A.C.P. Azevedo. 1983. Contribuição ao conhecimento da alimentação e do modo alimentar de serpentes do Brasil. Comunicações do Museu de Ciências da PUCRS, Porto Alegre, 26: 41-121.

LOPES, H.R.; C.F. RocHA \& A.S. ABE. 1991. Constricting behavior in snakes: is there a side dominance? Rev. Bras. Biol. 51 (4): 853-856.

Marques, O.A.V. \& G. Puorto. 1994. Dieta e comportamento alimentar de Erytrolamprus aesculapi, uma serpente ofiófaga. Rev. Bras. Biol. 54 (2): 253-259.

MCCOY, C.J. \& E. CENSKY. 1992. Biology of the yucatan hognosed pitviper, 
Porthidium Yucatanicum, p. 216-222. In: J.A. CAMPBELl \& E.D. BRODIE JR. (Eds). Biology of the Pitvipers. Arlington, Univ. Texas, 467p.

MoORI, A. 1991. Effects of prey size and type on prey handling behavior in Elaphe quadrivirgata. J. Herpetol. 25 (2): 160-166.

Mushinsiky, H.R. 1987. Foraging ecology, p. 302-334. In: R.A. SEIGEL; J.T. COLLINS; S.S. NOVAK (Eds). Snakes: ecology and evolutionary biology. New York, MacMillan Pubil. Co., 529p.

PUORTO, G.; I. LAPORTA-FERREIRA \& I. SAZIMA. 1991. Serpentes na selva de pedra. Ciência Hoje, Rio de Janeiro, 76 (13): 66-67.

SAzIMA, I. 1989. Comportamento alimentar da jararaca, Bothrops jararaca: encontros provocados na natureza. Cienc. Cult., São Paulo, 41: 500-505.

1992. Natural history of the jararaca pitviper, Bothrops jararaca, in southeastern Brazil, p.199-216. In: J.A. CAMPBELL \& E.D. BRODIE JR. (Eds). Biology of the Pitvipers. Arlington, Univ. Texas, 467p.

SAZIMA, I. \& A.S. ABE. 1991. Habits of five brazilian snakes with coral-snake patern, including a summary of defensive tatics. Stud. Neotrop. Fauna Environ. 26 (3): 159-164.

SAZIMA, I. \& A. J. ARGÔLO. 1994. Siphlophis pulcher (NCN). prey. Herp. Rev. 25 (3): 126.

SAZIMA, I. \& C.F.B. HADDAD. 1992. Répteis da Serra do Japi: Notas sobre história natural, p.212-236. In: L.P.C. MoRELlato (Ed.) História natural da Serra do Japi: Ecologia e preservação de uma área florestal no sudeste do Brasil. Campinas, UNICAMP \& FAPESP, $321 \mathrm{p}$.

SAZIMA, I. \& M. MARTins. 1990. Presas grandes e serpentes jovens: quando os olhos são maiores que a boca. Mem. Inst. Butantan 52 (3): 73-79.

SHINE, R. \& T. SCHWANER. 1985. Prey constriction by venomous snakes: a review and new data on Australian species. Copeia 4 (10): 1067-1071.

SHINE, R. \& J.K. WEBB. 1990. Natural history of australian typhloplid snakes. J. Herpetol. 24 (4): 357-356.

Strussmann, C. \& I. Sazima. 1993. The snake assemblage of the Pantanal at Poconé, Western Brazil: faunal composition and ecological summary. Stud. Neotrop. Fauna Environ. 28 (3): 157-168.

WILLARD, D.E. 1977. Constricting methods of snakes. Copeia 2: 379-382.

ZAher, H. \& U. CARAmaschi. 1992. Sur le statut taxinomique d'Oxyrhopus trigeminus et $O$. guibei (Serpentes, Xenodontinae). Bull. Mus. Natl. Hist. nat., Paris, 14: 805-827.

Recebido em 30.V.1995; aceito em 02.VIII.1996. 